\title{
Du français au créole. Phonétique, lexicologie et dialectologie antillaises, sous la direction de André
} THIBAULT

\section{Emanuela Cacchioli}

\section{(2) OpenEdition}

\section{Journals}

\section{Edizione digitale}

URL: http://journals.openedition.org/studifrancesi/16808

DOI: 10.4000/studifrancesi. 16808

ISSN: 2427-5856

\section{Editore}

Rosenberg \& Sellier

\section{Edizione cartacea}

Data di pubblicazione: 1 juillet 2019

Paginazione: 203-204

ISSN: 0039-2944

\section{Notizia bibliografica digitale}

Emanuela Cacchioli, «Du français au créole. Phonétique, lexicologie et dialectologie antillaises, sous la direction de André thiBauLt», Studi Francesi [Online], 187 (LXIII | I) | 2019, online dal 01 juillet 2019, consultato il 24 janvier 2021. URL: http://journals.openedition.org/studifrancesi/16808 ; DOI: https:// doi.org/10.4000/studifrancesi. 16808

Questo documento è stato generato automaticamente il 24 janvier 2021.

\section{(c) (i) () $९$}

Studi Francesi è distribuita con Licenza Creative Commons Attribuzione - Non commerciale - Non opere derivate 4.0 Internazionale. 


\title{
Du français au créole. Phonétique, lexicologie et dialectologie antillaises, sous la direction de André THIBAULT
}

\author{
Emanuela Cacchioli
}

\section{NOTIZIA}

Du français au créole. Phonétique, lexicologie et dialectologie antillaises, sous la direction de André тніваULt, Paris, Classiques Garnier, 2015, 506 pp.

1 Il volume curato da André thiBAult è una collettanea di contributi che intendono ampliare le conoscenze sul creolo da una prospettiva fonetica, lessicologica e dialettologica. Nel novembre del 2012 si è tenuto un convegno presso la Sorbona di Parigi con lo scopo di dare nuovo impulso alla ricerca sul creolo senza prendere necessariamente in considerazione la morfosintassi, settore al quale gli studiosi di tutto il mondo tendono a limitarsi. Thibault ripercorre velocemente, nell'introduzione, $\mathrm{i}$ titoli dei volumi che costituiscono un riferimento per la lessicologia storica, la lessicologia sincronica, la lessicografia, la dialettologia e la sociolinguistica. Per quanto sia possibile enumerare studi sul creolo in ogni ambito analizzato, il critico ravvisa la necessità di incentivare la ricerca per aggiornare, migliorare e potenziare le conoscenze che, a oggi, risultano ancora lacunose, imprecise o nebulose. I ricercatori che si sono riuniti a Parigi nel 2012 hanno intrapreso alcuni percorsi relativi in particolare alla fonetica, alla dialettologia e alla sociolinguistica storica.

2 La prima parte, focalizzata sulla fonetica, offre al lettore sei studi particolarmente interessanti. Annagret BoLlÉE, Éléments de phonétique diachronique des créoles français (pp. 17-42), prende in esame gli esiti dell'evoluzione dell'antica $h$ aspirata del francese, giungendo alla conclusione che i risultati sono legati a fattori molteplici e che la lettera $h$ utilizzata oggi può avere una diversa origine rispetto al francese. Con l'articolo Sur le lexique des français populaires maintenu dans le créole antillais (pp.43-98), Jean-Paul 
CHAUVEAU ripercorre i fenomeni che hanno dato origine alla formazione del lessico negli idiomi creoli. Un'analisi quantitativa condotta secondo tre assi (distratico, diacronico e diatopico) permette di contestare la teoria che sostiene la derivazione popolare da patois regionali francesi o da arcaismi provinciali per spiegare la presenza di numerosi vocaboli creoli. Secondo Chauveau, al contrario, si tratterebbe piuttosto di forme colte o di prestiti da linguaggi specialistici già in uso in epoca coloniale, poi reinterpretati $\mathrm{e}$ grammaticalizzati. Anche Marie-Christine HAZAËL-MASSIEUX (Sens figurés et métaphores. Pour le développement du lexique créole, pp. 99-127) si occupa di lessico e, in particolare, della necessità di un'analisi semantica più accurata per fornire al lettore una definizione corretta delle espressioni metaforiche. La studiosa analizza due esempi specifici: «kyòlòlò» e «tête-mabolo». Du français aux créoles - à travers la communication? Créolisation, délocutivité et métonymie pragmatique (pp. 129-168) è il titolo del contributo di Silke JANSEN e Ruth HOFFMANN. Le due ricercatrici riprendono il concetto di delocutività ovvero di metonimia pragmatica illustrato dai rappresentanti della linguistica cognitiva tedesca (Koch e Detges) e dimostrano che la nascita dei neologismi dovuti al processo di creolizzazione avviene in maniera nettamente minore rispetto a quanto sostenuto finora. L'articolo di Pierre RézeAu, Petit inventaire lexical de la correspondance du Bourguigon E. Berthot, directeur des Ponts et Chaussées de la Guadeloupe de 1843 à 1846 (pp. 169-201), è uno studio attento e puntuale dei fenomeni lessicali intervenuti in Guadalupa intorno alla metà del XVIII secolo, che si fonda sull'analisi di una corrispondenza privata, recentemente data alle stampe. Chiude la sezione, D'Albert le Grand à Makandal. Continuité et innovation dans le lexique de la magie et de la sorcellerie des créoles français (pp. 205-239) di Ulrike scholz. L'articolo verte su un'analisi del lessico relativo alla magia. Ogni unità semantica viene indagata da una prospettiva storicoetimologica, morfologica e etnologica.

3 La seconda sezione, dedicata alla dialettologia, contiene due contributi. Dialectologie historique dans la Caraibe. Textes anciens, données dialectales, hypothèses de travail (pp. 243-279) di Dominique fATTIER si concentra sul problema della localizzazione geografica del primo testo creolo conosciuto, Passion de Notre Seigneur selon Saint-Jean en langue nègre. L'analisi di alcuni lessemi e tratti grammaticali, confrontata con il materiale storico e contemporaneo a disposizione, induce la studiosa a ipotizzare un'origine nord-haitiana del testo. Jean LE DÛ e Guylaine BRUN-TRIGAUD, con L'Atlas Linguistique des Petites Antilles (ALPA). Premières approches géolinguistiques des aires lexicales, phonétiques et morphologiques (pp. 281-332), offrono uno studio approfondito delle varianti di alcuni vocaboli nelle diverse aree caraibiche in cui si parla il creolo. Il contributo risulta particolarmente interessante perché supportato da carte geolinguistiche in grado di rendere conto a livello visuale delle variazioni intercorse. Inoltre, vengono prese in considerazione anche aree non francofone come la Dominica, Santa Lucia, Trinidad e Oiapoque.

4 La terza parte si concentra sulla sociolinguistica storica. Jo-Anne S. FERREIRA, L'archivage et la préservation du patrimoine littéraire du créole français trinidadien. Vers la revitalisation (socio)linguistique (pp. 335-352), traccia una panoramica dettagliata del quadro storico e dell'evoluzione del creolo a Trinidad, degli sforzi di archiviazione della documentazione scritta e sonora disponibile e delle attività intraprese per assicurarne una rivitalizzazione linguistica. Con Les Grands Blancs de la Guadeloupe. Histoire des langues, sociolinguistique et phonologie (pp.353-424), Elissa PUSTKA si sofferma sul comportamento linguistico, complesso e paradossale, dei coloni francesi in Guadalupa. 
Oltre a individuarne la natura e i tratti caratteristici a livello fonologico e sociolinguistico, la studiosa ne analizza l'importanza e le funzioni legate agli scambi quotidiani. Albert VALDMAN, Du français colonial aux parlers créoles (pp. 425-460), prende in esame il francese coloniale e, tenendo conto dei dati storici e sociali che hanno accompagnato la nascita del creolo nei Caraibi, sostiene che esso abbia influenzato l'idioma locale sia a livello di struttura che di lessico e morfosintassi.

Il volume può essere annoverato tra gli studi di riferimento del creolo per la qualità dei contributi presentati e per aver saputo intercettare l'esigenza di nuovi percorsi di ricerca applicati agli idiomi creoli nati su base francese. Auspichiamo nuovi approfondimenti per potenziare le conoscenze di queste lingue sia a partire dal francese, come è avvenuto per i saggi qui presentati, che dagli altri molteplici influssi linguistici che hanno agito sull'evoluzione dei creoli. 